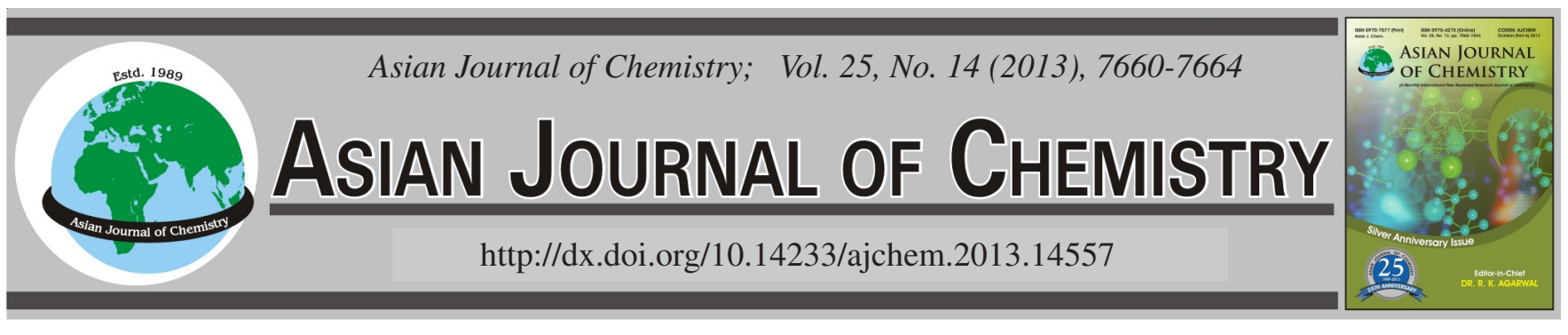

\title{
Amphoteric Modification of Sugarcane Bagasse Hemicelluloses and Characterization of the Novel Derivatives
}

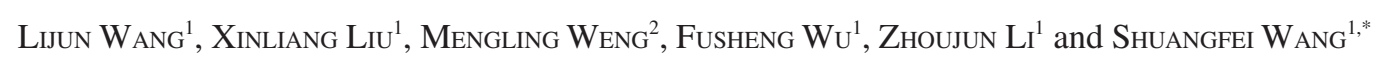

${ }^{1}$ Light Industry and Food Engineering Institute, Guangxi University, Nanning, P.R. China

${ }^{2}$ Guangxi Sugarcane Research Institute, Guangxi academy of Agricultural Science, Nanning, P.R. China

*Corresponding author: Fax:+86 771 3232874; Tel: +86 771 3236818; E-mail: wangs@gxu.edu.cn; wanglijun1103@163.com

(Received: 10 September 2012;

Accepted: 8 July 2013)

AJC-13782

\begin{abstract}
Modifiers were applied to make amphoteric modification on sugarcane bagasse hemicelluloses amount of modifiers and sodium hydroxide, reaction temperature, reaction time, the order of modification etc., would have some impact on the gender modification. Results showed that optimal cationic modified product could be obtained with $0.3 \mathrm{~g}$ (3-chloro-2-hydroxypropyl) trimethylammonium chloride and $0.1 \mathrm{~g}$ sodium hydroxide at $60^{\circ} \mathrm{C}$ for $2 \mathrm{~h}$; optimal anionic modified product could be obtained with $0.4 \mathrm{~g}$ chloro-acetic acid and $0.3 \mathrm{~g}$ sodium hydroxide at $80^{\circ} \mathrm{C}$ for $2 \mathrm{~h}$. Better gender modified products could be achieved as the order of anionic modification as the first. IR, NMR, scanning electronic microscopy and thermal analysis were used to characterize gender modified bagasse hemicelluloses. Amphoteric method in this study could draw carboxyl and quaternary ammonium into sugarcane bagasse hemicelluloses and molecular structure and morphology of sugarcane bagasse hemicelluloses after amphoteric modification were pointed out, with its thermal stability decreased.
\end{abstract}

Key Words: Sugarcane bagasse, Hemicellulose, Cationic modification, Anion modification.

\section{INTRODUCTION}

Sugarcane, being widely abundant in the nature and $c a$. 54 million tons bagasses of which have been generated all over the world, is a kind of renewable resource ${ }^{1}$. Sugarcane bagasses are commonly used for fuel, paper industry, manmade board, active carbon, dietary fibre, thermoplastics, biological diesel fuel and also for the production of ethanol, xylitol, protein-rich biological fertilizer, enzymes and amino acid $e t c .^{2-4}$. As the issue of energy saving and environmental protection has become hot in the world, therefore, the utilization of sugarcane bagasses seems a new strategy for preparing environmentally friendly biomaterials.

Cellulose, hemicellulose and lignin are the top three ingredients in sugarcane bagasse, with hemicellucose content being high up to $28-35 \%$. The utilization of hemicellulose has been aimed at preparing functional biopolymers such as cationic or anionic hemicelluloses because of the economical, biocompatible, non-toxic, biodegradable feather ${ }^{5,6}$. Chemical modification allows hemicelluloses having special properties which could broaden their applications.

It has been made of several different types of simple sugars, such as D-xylose, D-mannose, D-glucose, D-half of lactose, L-Arab sugar, 4-oxygen methyl-D-glucose hyaluronic acid and a few L-rat lee sugar, L-dec sugar etc. ${ }^{7,8}$. These sugars comprise the primary glycosyl of hemicellulose. Moreover, hemicellulose includes diverse types of polysaccharidee, such as xylan, xyloglucan and galactoglucomannan. To some extent, hemicellulose in sugarcane bagasse belongs to xylan ${ }^{9}$.

In recent years, hemicelluloses have been widely used in chemical industry, food and pharmaceutic preparation, paper making and coating, as it can be used for the raw material of gel $^{10-12}$, cationic polymer ${ }^{13}$ and even for the long chain alkyl ester derivatives ${ }^{14}$. The content of hemicellulose in pulp plays a critical role in the "beating" process and is a key factor affecting the quality of paper in paper industry, which involved with white degree and transparency and with an optical range from 30 to $50 \%$. Hemicellulose has a unique chemical structures, not only for the different types of monosaccharide or polysaccharide included, but also as the different types of functional groups, for example, hydroxyl acetyl, carboxyl, 7hydroxy etc. These different glycans process different chemical behaviour. However, the complexity of their structure does limit their application in industry which will eventually restrict their uses. Fortunately, these weaknesses can be overcome by chemical modification. Oxidation, hydrolysis, reduction, etherification, esterification and crosslinking reaction has been involved in this process ${ }^{15}$. And that provide us with necessary conditions to develop hemicelluloses unknown valuable characteristics ${ }^{16}$. 
In this study, gender modification was carried out on hemicelluloses extracted from sugarcane bagasse using modified agents and catalysts, with a result coming out that an amphoteric hemicelluloses was obtained under a certain condition. Infrared spectrum scanning, nuclear magnetic resonance spectroscopy and scanning electron microscope were used to figure out characterizations of the modified hemicelluloses and thermal stability was assayed by thermal analyzer simultaneously.

\section{EXPERIMENTAL}

Bagasse hemicellulose was obtained from Nanning Sugar Industry, first exposed against for a preparatory drying, then ground to pass a $0.7 \mathrm{~mm}$ size screen and finally dried in a cabinet oven for $16 \mathrm{~h}$ at $50{ }^{\circ} \mathrm{C}$. Other chemicals purchased were of analytical reagent grade.

Extraction process of hemicelluloses: The bleaching of sugarcane bagasse $\rightarrow$ delignification $\rightarrow$ adding the $\mathrm{NaOH}$ solution and extraction $\rightarrow$ centrifugation (fetching the supernatant $) \rightarrow$ adding acetic acid to $\mathrm{pH} 5.0 \rightarrow$ adding the $95 \%$ ethanol $\rightarrow$ centrifugation (fetching the precipitation) $\rightarrow$ drying for $24 \mathrm{~h}$ at the constant temperature of $50{ }^{\circ} \mathrm{C}$.

Cationic modification of sugarcane bagasse hemicelluloses: One gram dried sugarcane bagasse hemicellulose and certain amount of 3-chloro- 2-hydroxypropyl trimethyl ammonium chloride and sodium hydroxide, were placed in a three-neck flask, which fitted with isodynamic stirring devices and linked with a drying tube filled with calcium chloride. At a given temperature, the modifying reaction was carried out in the media of $95 \%$ ethanol. Filtered and washed reactant as soon as the end of reaction, then drying in the oven for $16 \mathrm{~h}$ at $50{ }^{\circ} \mathrm{C}$.

Anionic modification of sugarcane bagasse hemicelluloses: One gram dried sugarcane bagasse hemicelluloses, together with a certain amount of chloro-acetic acid and sodium hydroxide, were placed in a three-neck flask, which fitted with isodynamic stirring devices and linked with a drying tube filled with calcium chloride. At the certain temperature, the modifying reaction was carried out in the media of $95 \%$ ethanol. Filtered and washed reactant as soon as the end of reaction, then drying in the oven for $16 \mathrm{~h}$ at $50^{\circ} \mathrm{C}$.

Functional groups of cationic/anionic modification: The content of cationic functional groups (mmol-N $\left.+\left(\mathrm{CH}_{3}\right)_{3} / \mathrm{g}\right)$ and anionic functional groups (mmol-COOH/g) was expressed by Seghier et al. ${ }^{17}$ with modification.

Characterization of sugarcane bagasse hemicelluloses: The chemical structure of native hemicelluloses and derived hemicelluloses was evaluated by FT-IR, ${ }^{13} \mathrm{C}$ and ${ }^{1} \mathrm{H}$ NMR spectroscopies.

Liquid ${ }^{13} \mathrm{C}$ NMR spectrum chart was recorded at $75.4 \mathrm{MHz}$ with BrukerMSL-300 NMR equipment. $60 \mathrm{~g}$ of sample was dissolved in $0.75 \mathrm{~mL} \mathrm{D}_{2} \mathrm{O}$ at $25^{\circ} \mathrm{C}$, then were scanned for 30000 times.

Liquid ${ }^{1} \mathrm{H}$ NMR spectra were recorded with VARIAN 600 NMR spectrum at $600.24 \mathrm{MHz} .35 \mathrm{mg}$ sample was dissolved in the $0.6 \mathrm{~mL}$ DMSO, were then scanned for 64 times.

Infrared spectrum scanning detecting of modified samples were determined in BrukerTENSOR27 infrared spectrometer through $1 \%$ of the tablets $\mathrm{KBr}$ method.
Scanning electron microscope detection of the microstructure of samples was observed under the S-3400 N electronic scanning electron microscopy in 5000 times.

Thermal analysis was performed using thermogravimetric analysis (TGA) and differential thermal analysis (DTA) on a simultaneous thermal analyzer (DSC, USA). The apparatus was continually flushed with nitrogen. The sample was weighed between 9 and $11 \mathrm{mg}$ ans was heated from room temperature to $400{ }^{\circ} \mathrm{C}$, at a heating rate of $10^{\circ} \mathrm{C} / \mathrm{min}$.

Statistical analysis and experiment replicates: Data were analyzed by one-way analysis of variance (ANOVA) using the statistical software SPSS 11.5 for Windows (SPSS Inc., Chicago, IL, United States). All experiments were conducted in a completely randomized design with three replicates for each treatment.

\section{RESULTS AND DISCUSSION}

Effect of reaction conditions on functional group contents of modified hemicelluloses: The effect of 3-chloro2-hydroxyl propyl 3-methyl ammonium chloride on cationic poly-acryl modification of the sugarcane bagasse hemicellulose was carried at $60^{\circ} \mathrm{C}$ for $2 \mathrm{~h}$, with $1 \mathrm{~g}$ of sugarcane bagasse hemicellulose, $0.1 \mathrm{~g}$ of sodium hydroxide and quatity of 3chloro-2-hydroxyl propyl 3-methyl ammonium chloride varying as $0.1,0.2,0.3,0.4,0.5$ and $0.6 \mathrm{~g}$. Result was shown as Fig. 1 (upper panel). Effect of 1-chlorine acetic acid on anionic modification of the sugarcane bagasse hemicellulose was studied at $80{ }^{\circ} \mathrm{C}$ for $2 \mathrm{~h}$, with $1 \mathrm{~g}$ of sugarcane bagasse hemicellulose, $0.3 \mathrm{~g}$ of sodium hydroxide and quatity of 1-chlorine acetic acid varying as $0.1,0.2,0.3,0.4,0.5$ and 0.6 g. Result was shown as Fig. 1 (lower panel).

The function base's amount of cationic poly-acryl and anionic modified products both increased first and then decreased, while the modifier's amount increased. The optimal modifier's amount of cationic poly-acryl modification was $0.3 \mathrm{~g}$, while the optimal modifier's amount of anionic poly-acryl modification is $0.4 \mathrm{~g}$. That's because when the modifier's amount increased, the contact probability between modifier and bagasse hemicellulose increased, thus, the modified products function base's amount increased. In the reaction process, the modifier and sodium hydroxide reacted and formed epoxy compound first, then the modifier reacted with bagasse hemicelluloses, as a result, too more modifiers made epoxy compound and bagasse hemicelluloses's contact probability decreased, the modified products function base's amount decreased.

Effect of reaction conditions on functional group contents of modified hemicelluloses: The effect of sodium hydroxide on cationic poly-acryl modification of the sugarcane bagasse hemicellulose was studied at $60^{\circ} \mathrm{C}$ for $2 \mathrm{~h}$, with $1 \mathrm{~g}$ of sugarcane bagasse hemicellulose, the reaction time, $0.3 \mathrm{~g}$ of 3 chloro-2-hydroxyl propyl 3-methyl ammonium chloride and the amount of sodium hydroxide varying as $0.05,0.1,0.15$, $0.2,0.25$ and $0.3 \mathrm{~g}$.

The effect of sodium hydroxide on anionic modification of the sugarcane bagasse hemicellulose was studied at $80{ }^{\circ} \mathrm{C}$ for $2 \mathrm{~h}$, with $1 \mathrm{~g}$ of sugarcane bagasse hemicellulose, $0.4 \mathrm{~g}$ of 1-chlorine acetic acid for $0.4 \mathrm{~g}$ and the quatity of sodium hydroxide varying as $0.1,0.2,0.3,0.4,0.5$ and $0.6 \mathrm{~g}$. 

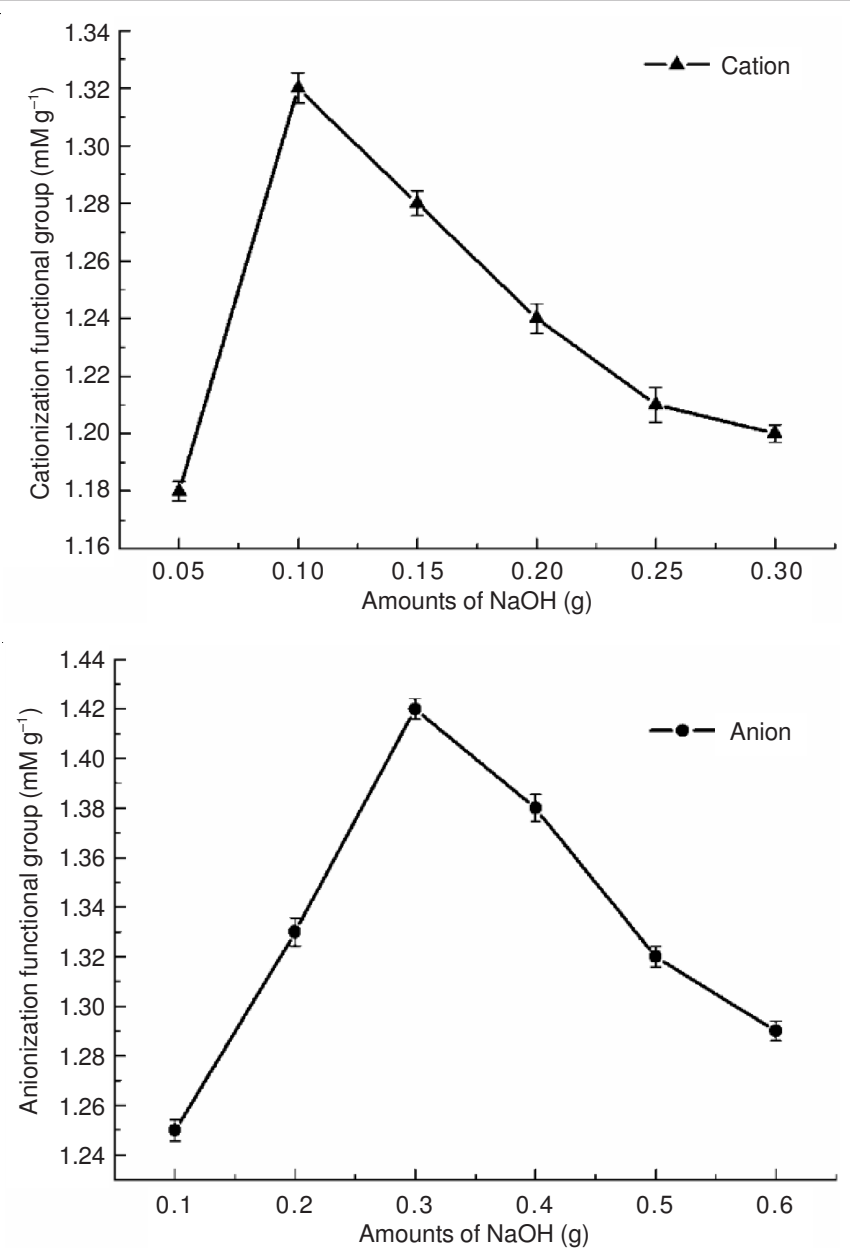

Fig. 1. Effect of the content of $\mathrm{NaOH}$ on the functional group contents of cationic/anionic modified products

The functional base amount of cationic poly-acryl and anionic modified products both increased first and then decreased, while the amount of sodium hydroxide increased. The optimal sodium hydroxide amount of cationic poly-acryl modification was $0.1 \mathrm{~g}$, while the optimal modifier amount of cationic poly-acryl modification was $0.3 \mathrm{~g}$. That's because the sugarcane bagasse hemicellulose was activated by sodium hydroxide during the reaction, so that close nuclear reactions happened and chitosan Ultrastructure's accessibility increased. When sodium hydroxide amount increased, the amount of the activated sugarcane bagasse hemicelluloses increased, so the functional base amount of modified products increased. But excess sodium hydroxide would cause side effects, which made the modifier decomposed in a high concentration alkaline environment, so that the modified products function base amount decreased.

Effect of reaction conditions on the modification: Sugarcane bagasse hemicelluloses' cationic poly-acryl and anionic modified reaction temperature was set at a range from room temperature to $100^{\circ} \mathrm{C}$ and time was set at a range from 0.5 to $10 \mathrm{~h}$. Reaction time would be longer if reactive temperature is lower, so the higher reactive temperature was adopted. However, too high temperature was adverse against bagasse hemicelluloses' alkalization. In this study, the sugarcane bagasse hemicelluloses of lower polymerization degree, alkalization and etherification can be conducted easily, we can choose higher reation temperature and appropriate reactive time. From the results, we can see the cationic poly-acryl modified reation's better reactive conditions are the reactive temperature of $60{ }^{\circ} \mathrm{C}$ for $2 \mathrm{~h}$ and the anionic modified reation's better reactive conditions are at $80{ }^{\circ} \mathrm{C}$ for $2 \mathrm{~h}$.

Effect of the modified order on function base amount of the modified products: There were two methods of the sugarcane bagasse hemicelluloses's amphoteric modification, cationization first and anionic modification followed or anionic modification first and cationization followed. In this work, we found cationization first benefited for the increasing of $\mathrm{N}$ $+\left(\mathrm{CH}_{3}\right)_{3}$ 's amounts and anionic modification first benefited for the increasing of-COOH's amounts. However, in terms of amphoteric modification function base's total amount $\mathrm{FG}=\mathrm{FGN}+\mathrm{FGC}, \mathrm{FG}$ will be higher when ationization first and then anionic modification proceed (Fig. 2).
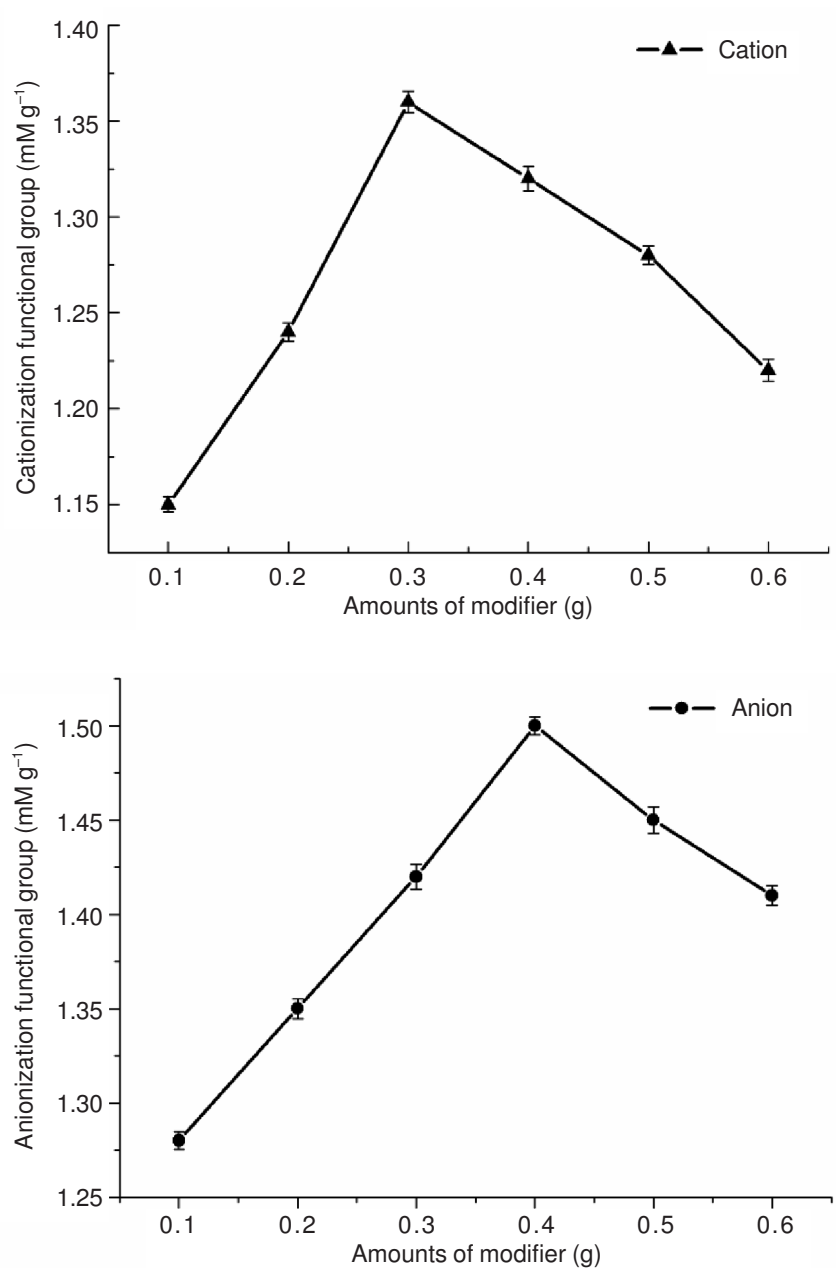

Fig. 2. Effect of the content of modifier on the functional group content of cationic/anionic modified products

FT-IR spectra detection: As can be seen in Fig. 3a, there was no peak between 1760 and $1680 \mathrm{~cm}^{-1}$, which showed that there was no carbonyl in the raw materials. While, carbonyl absorption peak and $\mathrm{C}-\mathrm{O}$ telescopic vibrating peak appear at 1722 and $1155 \mathrm{~cm}^{-1}$ could be seen in Fig. $3 \mathrm{~b}$ after modification. FT-IR spectra scanning confirmed the modification action between bagasse hemicelluloses and succinic anhydride, which suggested the carboxyl group was introduced. There was no 


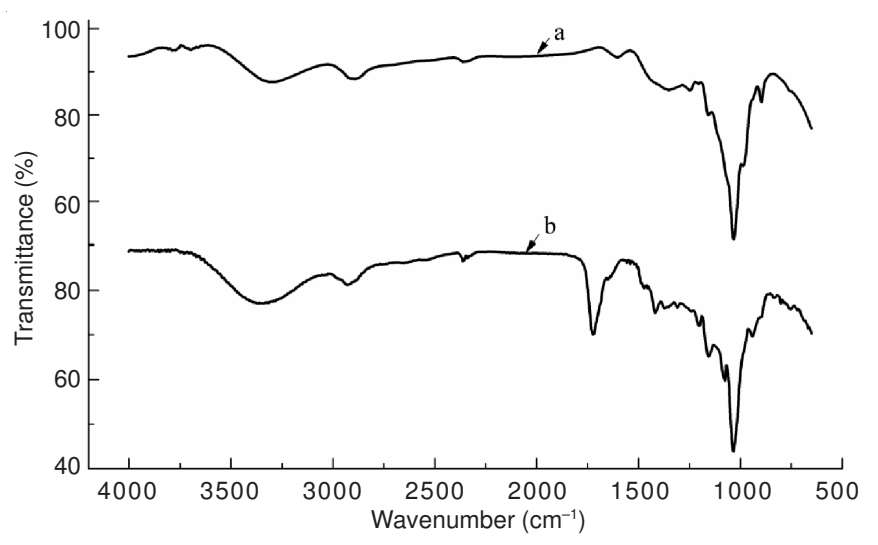

Fig. 3. FT-IR spectra of sugarcane bagasse hemicelluloses; (a) before modification; (b) after modification

peak between 1230 and $1030 \mathrm{~cm}^{-1}$ in Fig. 3a, which pointed out that few quaternary amine existed in raw material. C-N telescopic vibrating peak appears at $1070 \mathrm{~cm}^{-1}$ in Fig. $3 \mathrm{~b}$, which confirmed quaternary amine were introduced into sugarcane bagasse hemicelluloses.

${ }^{13}$ C NMR spectra detection: As shown as Fig. 4, carboxyl carbon signal peak at $173.98 \mathrm{ppm}$ could be observed in the modified hemicelluloses, with quaternary amine carbon signal peak at 29.24 ppm also being observed, while no carboxyl carbon and quaternary amine carbon signal peak could be observed in raw materials, suggesting the introduction of carboxyl and quaternary amine in sugarcane bagasse hemicelluloses.
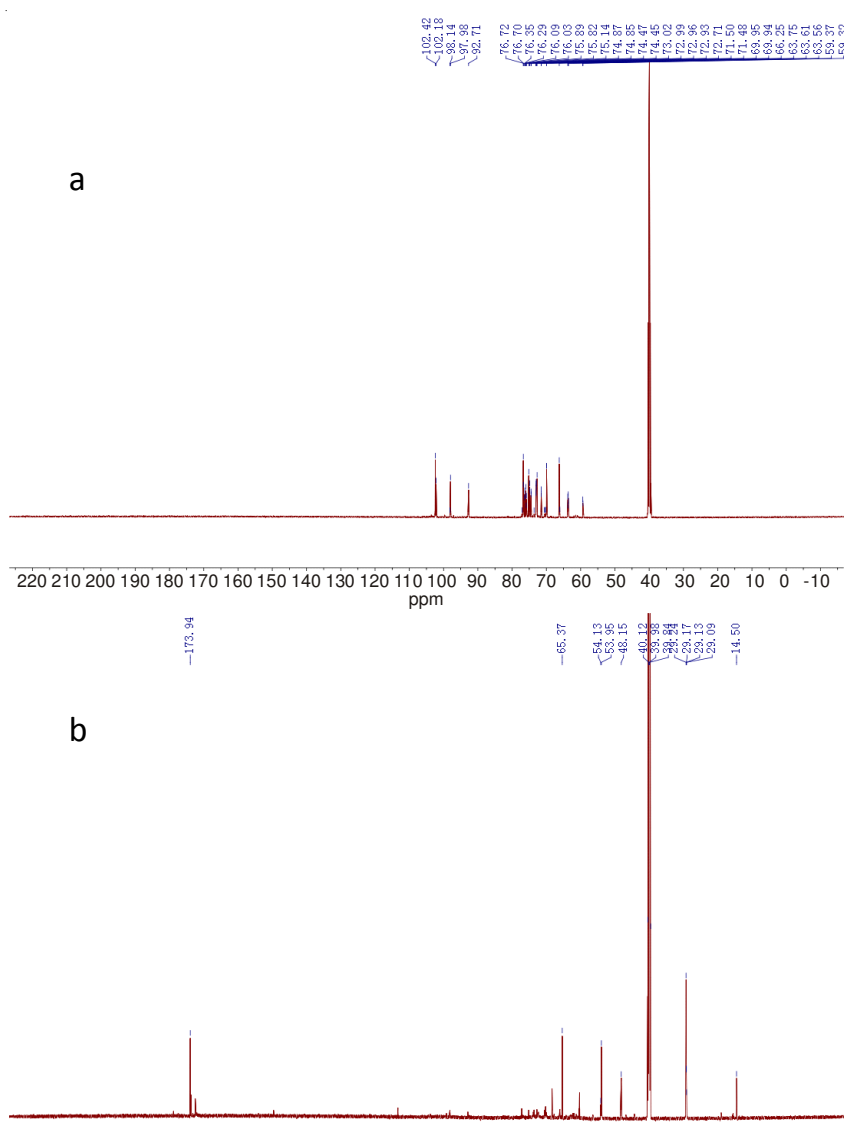

$22021020019018017016015014013012011010090 \quad 8070 \quad 6050 \quad 40302010 \quad 0^{\prime}-10$

Fig. 4. ${ }^{13} \mathrm{C}$ NMR spectrum of sugarcane bagasse hemicelluloses (a) before modification; (b) after modification
Scanning electron microscope: As can be seen from Fig. 5 , it was smoother in the surface of raw hemicelluloses and adsorption of microporous structure could be observed. While drape curly appears in the surface of the modified hemicelluloses, which suggested the increases of the surface area and it was prone to produce the adsorption of metal ions.
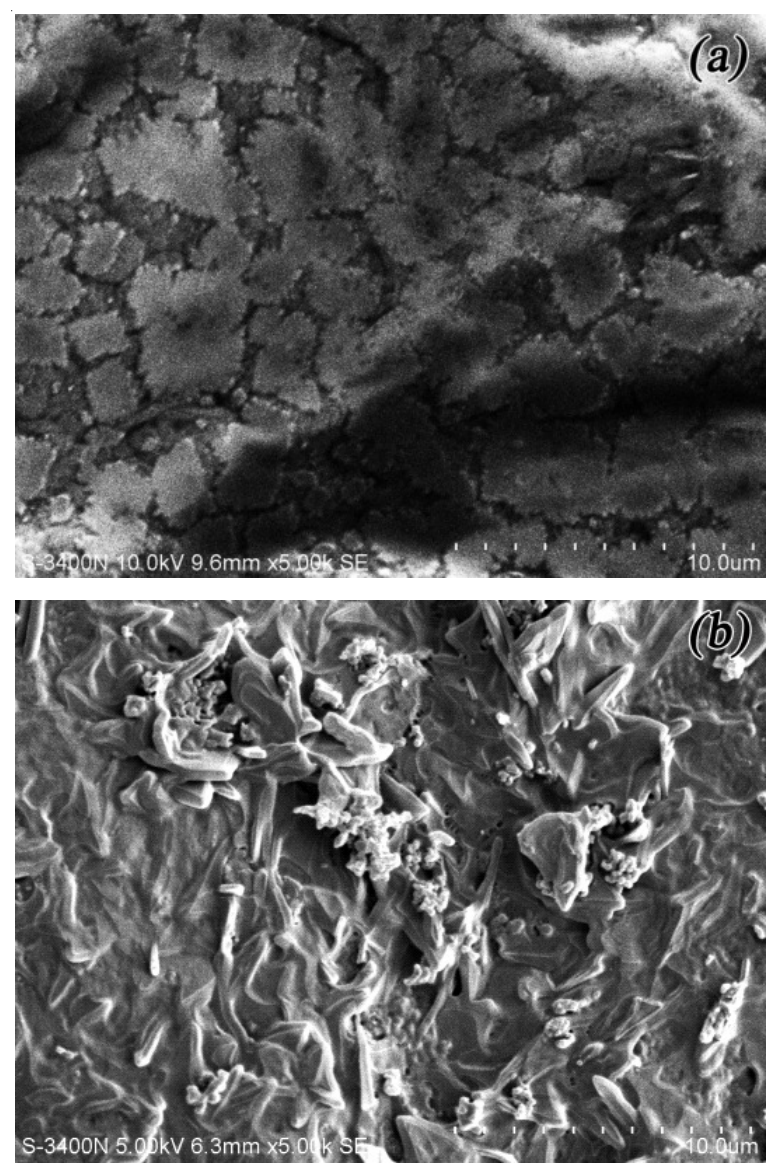

Fig. 5. SEM spectrum of sugarcane bagasse hemicelluloses $(\times 5000)$. (a- before modification; b-after modification)

Thermal analysis: As can be seen from the curve a in Fig. 6, the hemicelluloses's molten peak was at $345.65 \mathrm{~K}$ and the modified hemicelluloses had two molten peaks which were at 248.99 and $316.12 \mathrm{~K}$, respectively, which indicated that the successful introduction of carboxyl and quaternary amine in hemicelluloses. It also come out that the modified hemicelluloses showed lower thermal stability. Similar results were reported by Padadopoulos for acetylated Pinus sylvestris wood $^{18}$ and Severo for modified Pinus elliotii vae. Elliottii wood. According to the author, the wood presents an adverse effect on the modification because of its chemical composition.

\section{Conclusion}

Amphoteric modification of sugarcane bagasse hemicellulose was studied in this work. It can be pointed out that cationic poly-acryl modified hemicelluloses was easier to obtain from $1 \mathrm{~g}$ dried sugarcane bagasse hemicelluloses at $60^{\circ} \mathrm{C}$ for $2 \mathrm{~h}$ with $0.3 \mathrm{~g}$ of 3-chloro-2-hydroxyl propyl 3-methyl ammonium chloride and $0.1 \mathrm{~g}$ of sodium hydroxide, while, anionic modified hemicelluloses was easier to obtain from $1 \mathrm{~g}$ dried sugarcane bagasse hemicelluloses at $80{ }^{\circ} \mathrm{C}$ for $2 \mathrm{~h}$ with $0.4 \mathrm{~g}$ of 


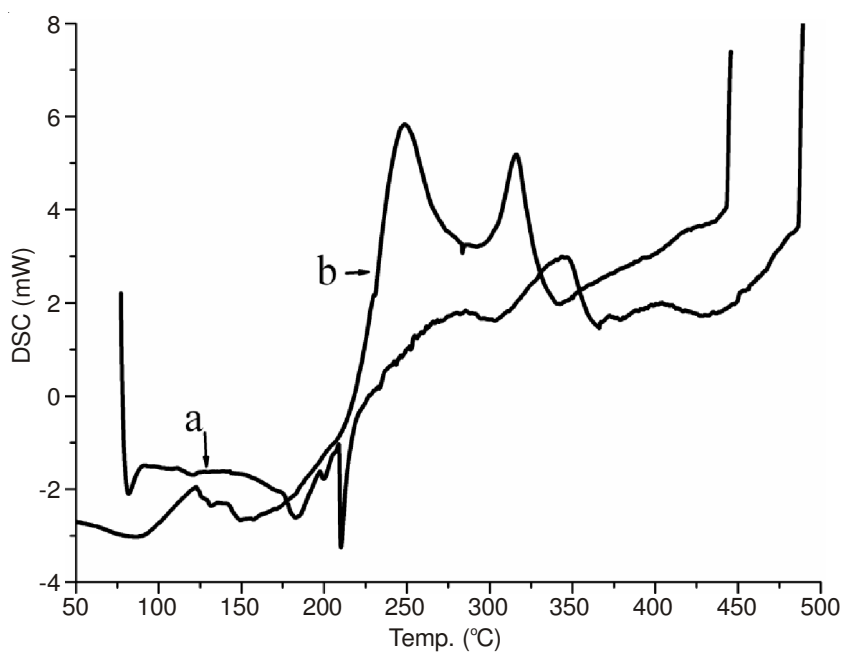

Fig. 6. Thermograms of sugarcane bagasse hemicelluloses. (a- before modification; b-after modification)

1-chlorine acetic acid and $0.3 \mathrm{~g}$ of sodium hydroxide. Better amphoteric modified products was produced when cationization first and anionic modification proceed followed. All samples were carried through FT-IR, nuclear magnetic resonance, thermal analyzer and scanning electron microscopy detection to characterize the characterizations. Results showed that the induction of carboxyl and quaternary amine under a certain modification method in sugarcane bagasse hemicelluloses. Structure and type of the sugarcane bagasse hemicelluloses were changed with amphoteric modification and the thermal stability of amphoteric modified sugarcane bagasse hemicelluloses was declined.

\section{ACKNOWLEDGEMENTS}

This work was financially supported by the National High Technology Research and Development Program (863 Program) (2009AA06A416) \& (2010GXNSFE 013006), National
Water Special Research Program (2008ZX07317-02-03A2, Guangxi Science Foundation for the Youth (0832005) and Research funds of The Guangxi Key Laboratory of Environmental Engineering (0804K016).

\section{REFERENCES}

1. R.C.L.B. Rodrigues, M.D.G.A. Felipe, J.B. Almeida E. Silva and M. Vitolo, Process Biochem., 38, 1231 (2003).

2. N.K. Amin, Desalination, 223, 152 (2008).

3. S.C. Rabelo, R.M. Filho and A.C. Costa, Appl. Biochem. Biotechnol., 153, 139 (2009).

4. M.A. da Cunha, A. Converti, J.C. Santos, S.T. Ferreira and S.S. Da Silva, Appl. Biochem. Biotechnol., 157, 527 (2009).

5. K. Schwikal, T. Heinze, B. Saake, J. Puls, A. Kaya and A.R. Esker, Cellulose, 18, 727 (2011).

6. X.W. Peng, J.L. Ren, L.X. Zhong, X.F. Cao and R.C. Sun, J. Agric. Food Chem., 59, 570 (2011).

7. X.F. Sun, R.C. Sun and J.X. Sun, J. Mater. Sci., 38, 3915 (2003)

8. R.M. Rowell and F.M. Keany, Wood Fiber Sci., 23, 15 (1991).

9. J.E. White, W.J. Catallo and B.L. Legendre, J. Anal. Appl. Pyrol., 91, 1 (2011).

10. I. Gabrielii and P. Gatenholm, J. Appl. Polym. Sci., 69, 1661 (1998).

11. I. Gabrielii, P. Gatenholm, W.G. Glasser, R.K. Jain and L. Kenne, Carbohydr. Polym., 43, 367 (2000).

12. M.S. Lindblad, E. Ranucci and A.C. Albertsson, Macromol. Rapid Commun., 22, 962 (2001).

13. A. Ebringerová, Z. Hromádková, M. Kacuráková and M. Antal, Carbohydr. Polym., 24, 301 (1994).

14. J.M. Fang, R. Sun, P. Fowler, J. Tomkinson and C.A.S. Hill, J. Appl. Polym. Sci., 74, 2301 (1999).

15. R. Sun, X.F. Sun and J. Tomkinson, In Hemicelluloses and their Derivatives, ACS Symposium Series, 2004-01-01, 2004; ACS Publications, pp. 2-23 (2004).

16. A. Ebringerová and T. Heinze, Macromol. Rapid Commun., 21, 542 (2000).

17. M.L. Seghier, F. Lazeyras, A.J. Pegna, J.M. Annoni and A. Khateb, Hum. Brain Mapp., 29, 461 (2008).

18. A.N. Papadopoulos, Eur. J. Wood Wood Prod., 64, 397 (2006). 